\title{
Donne, famiglie e pandemia. Tra vecchie e nuove discriminazioni al femminile
}

\author{
Francesca Dello Preite
}

\begin{abstract}
Il 2020 verrà ricordato come l'anno della pandemia da Covid-19, causata dal virus SARS$\mathrm{CoV}-2$ che repentinamente si è diffuso a livello planetario colpendo la salute e la vita di milioni di persone di ogni età e mettendo a dura prova gli assetti assistenziali, economici, scientifici e politici di tutti gli Stati che, con non pochi ostacoli e difficoltà, hanno cercato di dare risposte ai molteplici bisogni delle rispettive popolazioni. Il presente articolo, a partire da una riflessione sulle nuove povertà che il coronavirus ha prodotto nei contesti familiari, analizza le maggiori difficoltà vissute dalle donne durante il "confinamento" tra le mura di casa, dove i lavori domestici e la cura dei figli si sono moltiplicati, tanto sul piano qualitativo che quantitativo, e le violenze di genere hanno registrato un preoccupante aumento. Quanto successo in questi mesi è la tangibile riprova che le donne sono ancora oggi soggetti vulnerabili e che la strada per la parità sostanziale tra i generi continua a essere irta di ostacoli.
\end{abstract}

Parole chiave: crisi pandemica, nuove povertà, donne, lavoro di cura, violenza di genere.

\begin{abstract}
2020 will be remembered as the year of the Covid-19 pandemic, caused by SARS-CoV-2: a virus that suddenly spread worldwide affecting the health and the life of millions of people of all ages and putting a strain on welfare, economic, scientific and politic structures of all the Nations that have tried to respond to the multiple needs of their populations facing many obstacles and difficulties. Starting from a reflection about the new poverty lines that coronavirus produced in family context, this paper analyses the biggest difficulties that women faced during the "confinement" at home, when the housework and the children care multiplied, both in qualitative and in quantitative viewpoint, and gender violence registered an alarming increase. What happened in these months is a evident confirmation that women are still vulnerable people, and that the way to reach a significant gender equality is still fraught with obstacles.
\end{abstract}

Keywords: pandemic crisis, new poverties, women, care work, gender-based violence.

${ }^{1}$ Ricercatrice di Pedagogia generale e sociale presso il Dipartimento di Formazione, Lingue, Intercultura, Letterature e Psicologia (FORLILPSI) dell'Università degli Studi di Firenze. 


\section{Crisi pandemica e nuove povertà socio-familiari}

Il 2020, a seconda della prospettiva da cui lo si analizza, può apparire sia un anno scandito da interminabili giornate vissute tra le mura domestiche, senza avere la possibilità di incontrarsi con le altre persone sui luoghi di lavoro, a scuola o semplicemente camminando per strada, sia un anno caratterizzato da repentini cambiamenti sociali e scientifici che hanno modificato profondamente le nostre abitudini e le nostre esistenze. C'è chi ha paragonato questo frangente storico a "una guerra", chi lo ha definito "una tempesta", chi invece si è dissociato dall'uso di metafore belliche e catastrofiche, definendo errato mettere sullo stesso piano due fenomeni che si esplicano in azioni ben distinte, come quella del contrasto e della prevenzione (Battistelli, 2020). Al di là delle differenti interpretazioni che ciascuno vorrà attribuire ai mesi trascorsi tra un lockdown e l'altro, sta di fatto che l'emergenza sanitaria, nel suo protrarsi senza soluzione di continuità, ha intensificato le molteplici vulnerabilità sociali di cui conoscevamo tanto l'entità quanto l'urgenza (basti pensare all'annoso problema della disoccupazione giovanile), ma che in condizioni di "normalità" spesso cadevano nel cono d'ombra generato da scelte politiche ed economiche ritenute più impellenti e vantaggiose per il progresso del nostro Paese.

Prima dell'insorgere della pandemia, l'Istat (2020a) aveva rilevato che, in Italia, le famiglie in condizione di povertà assoluta erano circa 1,7 milioni (6,4\% del totale), per un numero complessivo di quasi 4,6 milioni di persone. A trovarsi in maggiore povertà assoluta erano in particolare le famiglie con un più alto numero di componenti, quelle con figli conviventi soprattutto se minori e le famiglie monogenitoriali, mentre nei nuclei in cui era presente almeno un anziano l'incidenza risultava più bassa. A tal proposito il report Le statistiche dell'Istat sulla povertà sottolinea che

in generale la povertà familiare presenta un andamento decrescente all'aumentare dell'età della persona di riferimento: le famiglie di giovani hanno più frequentemente minori capacità di spesa poiché dispongono di redditi mediamente più bassi e hanno minori risparmi accumulati nel corso della vita o beni ereditati (ivi, p. 3).

Cittadinanza e luogo di vita risultano variabili direttamente correlate alla povertà. Infatti, il documento dichiara che al Sud e nelle Isole l'incidenza delle famiglie in povertà assoluta era più alta che nelle altre aree 
territoriali del Paese (differenze riscontrabili anche a livello di singoli soggetti) e, per quanto riguarda lo stato di cittadinanza, viveva in povertà assoluta il 31,2\% delle famiglie straniere con minori contro il 6,3\% delle famiglie italiane con minori (cfr. ivi, in particolare p. 4).

Nel momento in cui veniva resa nota questa fotografia dell'Italia, seppur «il numero e la quota di famiglie in povertà assoluta [rimanesse] su livelli molto superiori rispetto a quelli precedenti la crisi economica del 2008-2009» (ivi, p. 1), vi erano comunque segnali di miglioramento.

La diminuzione della povertà assoluta si deve in gran parte al miglioramento, nel 2019, dei livelli di spesa delle famiglie meno abbienti [... . L'andamento positivo si è verificato in concomitanza dell'introduzione del Reddito di cittadinanza [... e ha interessato, nella seconda parte del 2019, oltre un milione di famiglie in difficoltà (ivi, p. 2).

Per alcuni mesi tali sviluppi hanno fatto sperare in un progressivo calo della povertà assoluta che potesse, in primo luogo, offrire opportunità di ripresa a favore delle nuove generazioni il cui ingresso nel mondo del lavoro è sempre più proteso in avanti con l'età come, del resto, la stessa idea di rendersi autonomi dai genitori e di costruirsi un proprio nucleo familiare. Ma con il dilagare della pandemia tale ottimismo non è durato a lungo tanto che sul finire del 2020 vari studi hanno denunciato un peggioramento dello status socio-economico dalla popolazione e delle famiglie italiane (e non solo), soprattutto, per chi già verteva in uno stato di precarietà e disagio.

Il dossier Gli anticorpi della solidarietà. Rapporto 2020 su povertà ed esclusione sociale in Italia (Caritas Italiana, 2020) analizzando gli effetti prodotti dal lockdown individua «il rischio di una grave recessione [...] che produrrà un impatto evidente sul benessere e la vita delle persone, favorendo anche la nascita di nuove forme di povertà» (ivi, p. 6). Prendendo in considerazione i dati raccolti presso i centri di ascolto delle sedi diocesane della Caritas emergono aspetti decisamente allarmanti:

da un anno all'altro l'incidenza dei "nuovi poveri" passa dal 31\% al 45\% (quasi la metà di chi si rivolge alla rete Caritas non lo aveva mai fatto in passato). Aumenta in particolare il peso delle famiglie con minori, delle donne, dei giovani, dei nuclei italiani e delle persone in età lavorativa. Accanto alla recrudescenza dei fenomeni di povertà già noti, si intravede, quindi, l'ipotesi di una nuova fase di "normalizzazione", come accaduto a seguito dello shock economico del 2008. Tuttavia lo scenario che si presenta oggi è decisamente peggiore rispetto ad allora, quando i poveri assoluti erano circa 1,7milioni (ivi, p. 6). 
Non sarà né facile né immediato uscire dal vortice creato dall'emergenza pandemica poiché, oltre ad affrontare i forti divari economici che si sono ampliati tra poveri e ricchi, sarà necessario intervenire sulle molteplici lacerazioni sociali, culturali, educative, affettive, emotive che questo tempo di incertezza e di precarietà ha prodotto a livello umano e globale. Come sostiene Edgar Morin nella sua recente opera scritta proprio in epoca di nuovo coronavirus, «l'avvenire imprevedibile è oggi in gestazione. Auspichiamo che sia per una rigenerazione della politica, per una protezione del pianeta e per un'umanizzazione della società: è tempo di cambiare strada» (2020, trad. it. 2020, p. 32).

\section{L'onda della crisi pandemica in ottica di genere}

Infanzia, giovani, donne, anziani/e e stranieri/e. È questo un elenco di agenti sociali che nel tempo abbiamo imparato a conoscere sotto più prospettive e dimensioni esistenziali e che, non a caso, vediamo riaffiorare nel dibattito pubblico soprattutto nei momenti di maggiore criticità. Simonetta Ulivieri, in riferimento alla storia dell'educazione, spiega che tali «categorie della differenza» (1994, p. 54) sono rimaste per secoli ai margini tanto della cultura ufficiale quanto del sentire comune così da passare inosservate e «sotto silenzio» (ibidem). Secondo la studiosa

i marginali rappresentano coloro che non hanno diritto di parola, gli esclusi dall'alfabeto, coloro che non fanno parte dei complessi disegni della classe dirigente e che vivono in condizioni di subalternità, di inferiorità, di indigenza, di diversità, che possono essere psicologiche, ma anche e soprattutto materiali (ivi, p. 57).

Non è un caso se in un periodo difficile come quello attuale bambini/e, ragazzi/e, donne, anziani/e italiani/e e straniere/i stiano pagando il prezzo più alto dell'emergenza. È questo il tangibile riscontro che i pregiudizi e gli stereotipi verso le categorie considerate più deboli e meno produttive per la ricchezza del Paese siano ancora ben radicati non solo nell'immaginario collettivo ma anche nelle logiche che guidano i sistemi istituzionali, politici e mediatici dei nostri giorni.

Con la chiusura dei servizi educativi 0-6 e delle scuole primarie, l'infanzia si è vista improvvisamente deprivata di tutti quegli spazi e di quei tempi in cui quotidianamente prendevano vita interventi di educazione e cura dichiarati, a livello nazionale e internazionale, una base essenziale 
per il buon esito dell'apprendimento permanente, dell'integrazione sociale e dello sviluppo personale (COM, 2013; Silva, 2017). In modo del tutto inaspettato e con non poche difficoltà metodologiche, contenutistiche e tecniche, l'intera progettualità educativa rivolta ai bambini e alle bambine è stata ricondotta ad attività di Didattica A Distanza (DAD) che in molti casi non sono riuscite a raggiungere tutti i contesti familiari per mancanza di strumenti digitali e/o per insufficienza di connessione alla rete, comportando un aggravio dei disagi soprattutto per i soggetti con disabilità, con bisogni educativi speciali, a rischio di povertà educativa e di esclusione sociale. Senza contare che tutto questo è avvenuto mettendo a dura prova anche i genitori, molti dei quali, "confinati" in casa in smartworking, si sono dovuti barcamenare tra i loro impegni lavorativi e la DAD dei figli. Bambine e bambini, proprio nell'età della vita caratterizzata dalla continua e fondamentale scoperta del mondo attraverso l'interazione e la relazione con l'altro/a da sé, hanno visto venir meno la dimensione conoscitiva ed esplorativa che accade negli spazi e nei luoghi extradomestici dove si apprende quel vivere in comunità che crea dialogo e confronto aprendo lo sguardo alle alterità che fanno crescere.

Eppure, poche e poco ascoltate sono state le voci che si sono levate durante il lockdown in difesa dei diritti dell'infanzia, per rimettere al centro della discussione pubblica le esigenze educative dei più piccoli che costituiscono il nostro futuro (Ammaniti, 2020). Come ha affermato Emiliano Macinai in un recente articolo apparso sulla Repubblica:

l'infanzia continua ad essere $[\ldots]$ quello che per molti versi è sempre stata da quando, per usare le parole di Philippe Ariès, è stata scoperta: una condizione che penalizza un intero gruppo di popolazione, un'intera classe di cittadini. Finché i diritti saranno ordinati secondo priorità, vi sarà sempre un soggetto collettivo posto in una condizione di subalternità (20.11.2020, s.p.).

Un'altra narrazione, per molti versi analoga alla precedente ma per altri connotata di sue specifiche peculiarità, è quella relativa all'adolescenza, anch'essa piombata da un giorno all'altro nella "solitudine" di giornate trascorse nelle proprie stanze senza poter contare di ritrovarsi con i pari nei quotidiani luoghi dell'incontro dove si sperimentano le prime esperienze amicali, amorose e, soprattutto, ci si confronta sulle identità in divenire (Mancaniello, 2020; Palmonari, 2001; Vegetti Finzi, Battistin, 2000). In questi mesi, ragazze e ragazzi hanno cercato di "riempire" questi loro "nuovi tempi liberi" con navigazioni, conversazioni 
e incontri virtuali, resi possibili da quell'uso "disinvolto" delle nuove tecnologie che li contraddistingue come "nativi digitali". Non sappiamo ancora esattamente quali saranno gli esiti di queste interminabili giornate che gli/le adolescenti hanno passato immersi/e nel web, ma alcune avvisaglie già si stanno palesando e le possiamo cogliere nei reiterati attacchi di cyberbullismo volti a denigrare l'integrità identitaria di chi non segue i canoni performanti del gruppo, negli hate speech carichi di odio verso ebrei, stranieri, omosessuali, nei fenomeni del revenge porn, del sexting, del cyber-harassment (per citarne solo alcuni), tutte forme di violenza che in questo periodo di sovraesposizione alla rete hanno fatto crescere tra i/le giovani sia le vittime che i carnefici.

Anche analizzando gli effetti del lockdown in chiave di genere ne emerge un quadro altrettanto preoccupante (Brambilla, 2020; D'Addio, 2020; Dugarova, 2020; EIGE, 2021). Le donne, in questi mesi, tanto in campo lavorativo che familiare, hanno visto repentinamente minata una buona parte di quei diritti acquisiti negli ultimi decenni mediante azioni politico-sociali che hanno dovuto, in prima istanza, fare i conti con una cultura patriarcale fortemente radicata (Cagnolati, Pinto Minerva, Ulivieri, 2013; Cantarella, 2019; Priulla, 2013) che ancora oggi non permette all'universo femminile di godere pienamente di pari opportunità in tutti gli ambiti di vita (Caraveo, 2017; Columba, 2018; Lopez, 2017; Musi, 2019).

A dimostrazione di tutto ciò si considerino i recenti dati che l'Istat ha pubblicato sui tassi di occupazione, disoccupazione e inattività relativi al mese di dicembre 2020, da cui si evince che, in termini assoluti, rispetto ai 101 mila posti di lavoro venuti a mancare, 99 mila sono stati persi dalle donne (Istat, 2021a). Gian Carlo Blangiardo, presidente dell'Istat, durante una recente audizione alla Camera dei Deputati ha tracciato una situazione più che incerta sulla condizione lavorativa femminile:

nel terzo trimestre 2020, il calo occupazionale tendenziale tra le donne pari a $-3,5 \%$ contro il $-2 \%$ degli uomini - diventa ancora più marcato se si tratta di donne al di sotto dei 35 anni di età $[\ldots]$ o residenti nel Mezzogiono [...]. Le donne occupate con contratto a tempo determinato, tra il terzo trimestre 2019 e il terzo trimestre 2020 , sono diminuite del $16,2 \%$, rispetto a un valore che per gli uomini si è fermato al 12,4\%. L'occupazione femminile ha inoltre mostrato diminuzioni più marcate, rispetto agli uomini, soprattutto per le attività del settore degli alberghi e ristoranti [...] e del settore dei servizi alle famiglie [...] dove la componente femminile rappresenta l' $87 \%$ dell'occupazione del settore. Infine, anche le lavoratrici straniere sembrano aver subito gli effetti più marcati: rispetto al terzo trimestre 2019 hanno registrato una 
diminuzione del 9,1\%, a fronte di un calo pari al 3,8\% tra i lavoratori stranieri maschi (Istat, 2021b, p. 10).

È evidente che in questa congiuntura il problema della segregazione orizzontale (Iori, 2014; Sartori, 2009; Schimmenti, 2005) abbia doppiamente penalizzato le lavoratrici. Infatti, come evidenziano i dati, sono stati soprattutto i servizi alle famiglie, la ristorazione, il piccolo commercio, comparti a prevalenza femminile, ad aver subito le maggiori restrizioni con lunghe e ripetute chiusure che in molti casi sono divenute definitive con la conseguente perdita dei posti di lavoro.

Se quanto descritto rappresenta solo un breve spaccato dello scenario che ha preso forma in campo lavorativo, cosa è accaduto, invece, tra le mura domestiche? In che modo le donne, ed in particolare le madri, hanno ri-configurato i propri tempi di vita, le relazioni intrafamiliari, $\mathrm{i}$ ruoli legati alla cura dei/delle figli/e e al lavoro domestico?

Partiamo da una breve premessa. Come gli studi di genere più volte hanno dimostrato, nonostante all'interno delle famiglie contemporanee vi siano stati importanti cambiamenti nei rapporti tra i partner (Contini, Ulivieri, 2010; Marone, 2016; Saraceno, 2016, 2017), le donne continuano a dedicare ai lavori domestici e alla cura dei figli molte più ore rispetto ai loro compagni o mariti e questo anche quando hanno un impiego extradomestico. Alessandra Gigli afferma che, nonostante all'interno delle coppie attuali le relazioni siano divenute più simmetriche rispetto al passato, le donne, paradossalmente, hanno «guadagnato un carico di lavoro di gran lunga superiore a quello maschile» e, anche per questo, le definisce «madri "acrobatiche" [...]: abili organizzatrici, contemporaneamente efficaci in molti ambiti, con un impiego giornaliero superiore a quello dei partner; ma anche donne affaticate e, in alcuni casi, sempre sull'orlo di una crisi di nervi» (2010, p. 93).

La pandemia, purtroppo, non ha migliorato lo status quo descritto dalla studiosa. Secondo la ricerca Prima, durante e dopo il Covid: disuguaglianze in famiglia, condotta da Episteme, con interviste CAWI (Del Boca, Oggero, Profeta, Rossi, Villosio, 2020), con la chiusura dei nidi d'infanzia e delle scuole sia il lavoro domestico che l'accudimento dei figli sono notevolmente aumentati. Diversamente dai tempi pre-Covid, non è stato possibile condividere questo incremento dei carichi familiari con le nonne e i nonni che nel nostro Paese rappresentano una grande risorsa per la cura dei nipoti (Di Sandro, 2020). La ricerca, avviata nel 2019 su un campione di 1250 donne occupate, rivolgendo alle partecipanti alcuni quesiti relativi ai partner, ha permesso al team di indagare 
se il lockdown abbia generato dei cambiamenti all'interno della coppia sia in riferimento al lavoro domestico che alla cura dei figli. I dati analizzati evidenziano che il $68 \%$ delle partecipanti ha dedicato più tempo di prima al lavoro domestico, il 29\% lo stesso tempo e solo il 3\% meno di prima, mentre per quanto riguarda le risposte relative ai partner si evince che il $55 \%$ di questi non ha modificato per niente il proprio tempo, il $6 \%$ lo ha diminuito e solo il $40 \%$ ne ha dedicato di più.

Passando alla cura dei figli, mentre le madri confermano sostanzialmente i valori registrati per il lavoro domestico, i padri sembrano aver compiuto qualche progresso in più. La ricerca infatti mostra che il $61 \%$ delle madri lavoratrici ha visto crescere l'impegno verso la cura dei figli, il 34\% ha mantenuto gli standard precedenti e solo il 5\% li ha ridotti; per i partner invece si nota che il $51 \%$ ha aumentato il tempo a disposizione per i figli, il $45 \%$ non ha cambiato le proprie abitudini e solo il $3 \%$ ha ridotto tale impegno.

Seppur non distribuiti equamente tra madri e padri, i risultati ottenuti dall'indagine mostrano che il lockdown ha comunque influenzato gli assetti familiari vedendo crescere, per quanto attiene il genere maschile, soprattutto l'impegno verso la cura dei figli, una dimensione che, non a caso, già da alcuni anni appare in trasformazione nei giovani padri. Come si evince infatti dalla letteratura (Contini, 2010; Murgia, Poggio, 2011; Perillo, 2016; Zanatta, 2011), anche in periodi di "normalità", è sempre più frequente vedere i padri trascorrere il proprio tempo con i figli in diverse attività, da quelle ludiche e di svago a quelle relative alla scuola e alla salute, e questa tendenza appare più marcata quando la compagna ha un'occupazione lavorativa, nei Paesi dove il tasso di occupazione delle madri è elevato e nei padri con un migliore grado d'istruzione (Saraceno, 2016, p. 103). Ma il maggior tempo dedicato ai/ alle figli/e è solo un piccolo tassello di un mosaico molto ampio e complesso la cui realizzazione richiede, in primis, la messa in discussione di quelle barriere culturali che continuano ad ostacolare la piena condivisione delle responsabilità e dei carichi familiari all'interno delle coppie. Si tratta di una impasse che per essere affrontata nella sua complessità ha bisogno di un progetto trans-formativo ampio, intenzionale ed ambizioso che, oltre a stanziare maggiori risorse in campo sociale ed economico (risultate molto limitate e parziali durante la pandemia), miri a un investimento educativo e formativo che interessi tutte le età della vita attraverso cui sollecitare e promuovere processi capaci di generare nuovi modi di pensare, di costruire e di vivere le relazioni, tra le persone e tra i generi (Loiodice, 2020). 


\section{Vite interrotte dalla violenza domestica}

Un altro vulnus che non ha risparmiato le donne durante il distanziamento per il coronavirus è stata la violenza di genere subita nelle sue diverse configurazioni: da quella psicologica e fisica a quella economica e sessuale. Pochi giorni prima che venisse dichiarato lo stato di emergenza sanitaria e che scattassero le misure per il contenimento dei contagi, la Commissione Europea aveva presentato ai principali Organi dell'Unione la nuova strategia per la parità di genere 2020-2025, indirizzo politico che, riconoscendo nella promozione della parità tra donne e uomini un valore cardine e un compito precipuo dell'UE, indica come primo obiettivo da raggiungere in tutti gli Stati membri quello di porre fine alla violenza di genere, una piaga dalle dimensioni sproporzionate su cui «si continua a tacere e a chiudere gli occhi» (COM, 2020, pp. 1-3).

Nella prima parte del documento la Commissione dichiara che donne e uomini dovrebbero godere equamente della piena libertà di esprimere i propri sentimenti, le proprie idee e le proprie scelte di vita senza subire le limitazioni imposte da ruoli di genere stereotipati.

Chiunque dovrebbe essere al sicuro nella propria casa, nelle relazioni più strette, sui luoghi di lavoro, negli spazi pubblici e online. Le donne e gli uomini, le ragazze e i ragazzi, in tutta la loro diversità, dovrebbero essere liberi di esprimere le loro idee e le loro emozioni e di perseguire le loro scelte formative e professionali senza sentirsi vincolati da ruoli di genere stereotipati (COM, 2020, p. 3).

Ma dal piano dei principi e dei valori a quello della realtà il passo non è breve. Sono ancora troppi coloro che violano la parità tra uomini e donne ricorrendo a forme di odio in chiave sessista e ostacolando qualunque azione contro la violenza e la parità di genere. Secondo le stime della European Union Agency for Fundamental Rights (FRA, 2014) in Europa i livelli delle violenze di genere sono molto critici: il 55\% delle donne ha subito molestie sessuali, il 33\% violenze fisiche e/o sessuali; il $22 \%$ violenze ad opera del proprio partner (COM, 2020, p. 3).

Il fenomeno non riguarda solo specifiche categorie di donne e determinati contesti ma risulta trasversale a tutte le età, a tutti i ceti sociali e a tutti gli ambiti di vita sia pubblici che privati. La strategia dichiara, quindi, che la violenza di genere è «una delle maggiori sfide delle nostre società» e che va contrastata sostenendo e proteggendo le vittime e facendo sì che i responsabili rispondano dei loro comportamenti violenti. 
A distanza di qualche giorno dalla presentazione della strategia 20202025 , mentre il virus paralizzava di ora in ora le attività economiche e la mobilità delle persone, non è riuscito con altrettanta efficacia a sfrenare la violenza su tutte quelle bambine, ragazze e donne che, "rinchiuse" in casa con i loro stessi carnefici, hanno percepito e visto crescere il rischio per la propria incolumità. Da tempo gli studi sulla violenza di genere (Cirillo, 2018; Dello Preite, 2019; Lipperini, Murgia, 2013; Loiodice, 2020; Ulivieri, 2014) attestano che il nucleo familiare si configura come il luogo più pericoloso per le donne (e quando presenti per i/le loro figli/e) soprattutto in relazione a quelle condotte violente che il Codice penale definisce «maltrattamenti contro familiari o conviventi» (art. 572). È, infatti, proprio tra le pareti domestiche, luogo per antonomasia degli affetti, della sicurezza e della protezione, che gran parte delle vittime inizia ad essere controllata, offesa, svalorizzata, picchiata e, nei casi più estremi, uccisa dal proprio partner che non ne accetta in alcuna forma l'autonomia, l'indipendenza, la libertà.

Cosa è accaduto nel nostro Paese durante la pandemia? Che effetti ha prodotto sulle donne vittime di violenza domestica la prescrizione di rimanere a casa con il limite di uscire solo per i bisogni di prima necessità? Di primo acchito, se ci si fermasse ad analizzare esclusivamente i numeri delle denunce presentate alle Forze dell'ordine, degli accessi fisici delle donne ai centri antiviolenza (CAV) e dei femminicidi, fattispecie che a marzo 2020 hanno segnato un calo rispetto allo stesso periodo degli anni precedenti (Istat, 2020b), si potrebbe cadere nell'equivoco di credere che lo stare a casa, limitando le uscite e i contatti con l'esterno, abbia salvaguardato le donne dalla violenza. Questa interpretazione, però, ridarebbe forza all'ideologia che reputa le donne soggetti deboli, destinate per natura alla sfera privata piuttosto che alla vita pubblica inadeguata alle loro caratteristiche fisiche, cognitive ed affettive. Sulla base di studi condotti sulla violenza di genere dopo eventi di calamità naturali o in tempi di crisi possiamo, invece, scartare questa ipotesi gender-blind e sessista. Infatti, le evidenze raccolte dimostrano che in tali periodi l'accesso ai servizi di supporto diventa più difficile per le vittime a causa o della chiusura degli stessi servizi, o per sotto-finanziamenti, o per l'introduzione di nuove norme che ne limitano la fruizione (cfr. EIGE, 2021, in particolare p. 11).

Come ha dichiarato la Senatrice Valeria Valente nell'ambito della Commissione Parlamentare d'Inchiesta sul Femminicidio, nonché su ogni forma di violenza di genere, svoltasi il 26 marzo 2020 presso il Senato della Repubblica, 
la riduzione dei contatti esterni e la prolungata condivisione degli spazi domestici con il partner maltrattante rendono ancora più difficile l'emersione di situazioni di violenza domestica e assistita: si è registrato [...] nelle ultime settimane una diminuzione non solo degli accessi fisici delle donne ai centri antiviolenza e agli sportelli, ma anche delle stesse denunce per maltrattamenti. [...] Il calo delle denunce [...] e degli accessi non significano purtroppo che la violenza contro le donne sia in regressione, ma sono invece il segnale di una situazione nella quale le donne vittime di violenza rischiano di trovarsi ancora più esposte alla possibilità di controllo e all'aggressività del partner maltrattante. Per far fronte a questa emergenza nell'emergenza il Parlamento e il Governo devono predisporre misure e risorse economiche aggiuntive e procedure più snelle per garantire misure di protezione, sostegno e accoglienza alle donne e ai minori coinvolti, assicurando in particolare l'operatività [...] delle strutture antiviolenza (Senato della Repubblica, 2020, p. 3)2.

Il tempo, infatti, ha dato ragione a chi non si è lasciato trarre in inganno dai primi riscontri quantitativi. Uno strumento che si è rivelato di massima utilità per raccogliere le richieste di aiuto e monitorare l'andamento e la gravità delle violenze domestiche è stato il numero verde istituito dal Dipartimento delle Pari Opportunità della Presidenza del Consiglio rimasto attivo 24 ore su 24. Tra marzo e ottobre 2020 sia le telefonate che i messaggi ricevuti tramite chat hanno avuto un incremento pari al 71,7\% rispetto allo stesso periodo del 2019. Sono sostanzialmente raddoppiate le domande di aiuto da parte delle vittime e le segnalazioni per casi di violenza, problematiche che unitamente hanno rappresentato il $45,8 \%$ delle chiamate valide e che rispetto all'anno precedente sono aumentate del 107\% (Istat, 2020c). La stragrande maggioranza delle donne che ha contattato il servizio ha dichiarato che le violenze subite non erano episodi sporadici bensì comportamenti reiterati nel tempo (anche da anni) che, in quel momento, generavano in loro forti preoccupazioni per la propria incolumità (e quella dei/delle figli/e se presenti) e paura di morire. Nonostante le istituzioni abbiano cercato di adottare una serie di misure urgenti che potessero, quanto meno, contenere i danni di questa barbarie (sostegni economici ai CAV e alle Case rifugio, campagne social a sostegno delle donne, azioni di sensibilizzazione, ecc.), le strategie della "rincorsa ai ripari" adottata in questi mesi o quella del "tirare al risparmio", che da anni accompagna il fenomeno della violenza contro le donne, non sono più ammissibili. Le violenze avvenute e aggravate

${ }^{2}$ Per maggiori dettagli si rimanda ai Riferimenti bibliografici, N.d.R. 
dalla pandemia, a lungo taciute o considerate di secondo ordine, hanno oggi bisogno di essere denunciate, conosciute e, soprattutto, affrontate mediante un piano integrato d'interventi a breve, medio e lungo termine che a partire da azioni mirate ad aiutare le donne ad uscire dalla violen$\mathrm{za}$, si rivolga contemporaneamente a tutti i cittadini e le cittadine, ed in particolare alle nuove generazioni, con proposte educative formali, informali e non formali volte a comprendere come, quando e perché le relazioni tra i generi assumono forme e toni violenti e quali processi possano sostenere donne e uomini di tutte le età a costruire rapporti interpersonali basati sul rispetto reciproco e la valorizzazione di ciascuno.

\section{Da dove ri-partire?}

Non sarà affatto scontato rielaborare le narrazioni e le immagini che per un intero anno hanno scandito (e probabilmente così sarà anche nei prossimi mesi) le nostre giornate, presentandoci notizie agghiaccianti provenienti da ogni angolo della Terra. La pandemia da Covid-19 resterà a lungo impressa nei nostri ricordi e i suoi effetti sulla società, probabilmente, non avranno breve durata, soprattutto per quelle persone che il coronavirus ha reso più povere e ha lasciato nella solitudine.

Non dobbiamo però sprecare il tempo vissuto e i molti sacrifici affrontati restando nell'immobilismo e nello scoraggiamento. È necessario capovolgere questa medaglia per interpretare il 2020 anche come un anno di inatteso avanzamento scientifico promosso dall'incessante lavoro di studiose e studiosi di ogni disciplina (da quelle biomediche a quelle umanistiche) che senza soluzione di continuità si sono applicate/i nella ricerca per generare nuove idee e nuove risorse volte a sostenere il funzionamento di tutti quei sistemi strettamente collegati all'agire umano. Adesso diventa fondamentale trasformare questa inedita esperienza in una sfida di sviluppo sostenibile, guardando al futuro con la consapevolezza che il mondo che intendiamo consegnare alle cittadine e ai cittadini di domani dipende dalle decisioni che prendiamo oggi. Questo vale anche per le questioni di genere tuttora pervase da diseguaglianze, discriminazioni e violenze che le donne subiscono ogni volta che decidono di infrangere gli schemi imposti dal modello eteronormativo (Burgio, 2019) per intraprendere traiettorie esistenziali che diano valore ai loro talenti (Dello Preite, 2018; Margiotta, 2018).

La Pedagogia può rispondere a queste istanze, urgenti e improcrastinabili, con un impegno militante (Tomarchio, Ulivieri, 2015) e inten- 
zionale che promuova processi educativi e formativi volti a sviluppare menti critiche e riflessive capaci di sollecitare il soggetto-persona a porsi domande, a valutare i fatti da più angolazioni, a comprendere i rischi che certe azioni sessiste potrebbero causare alla propria vita e a quella degli/ delle altri/e. Si tratta, quindi, direbbe Franco Cambi, di compiere «una rivoluzione radicale, profondamente innovatrice, anche inquietante [...] in quanto spiazza abitudini, tradizioni, formae mentis diffuse e legittimate e promuove un fascio di disposizioni e atteggiamenti del tutto (o fondamentalmente) altri» (2001, p. 29).

Questa può diventare la strada per ri-equilibrare i rapporti intergenere; ma per un vero cambiamento non bastano le pure intenzioni: serve il contributo di tutti e di tutte su più fronti, da quello politico ed economico, a quello culturale e sociale.

\section{Riferimenti bibliografici}

Ammaniti M. (2020): E poi, i bambini. I nostri figli al tempo del coronavirus. Milano: Solferino.

Battistelli F. (2020): Coronavirus: metafore di guerra e confusione di concetti. MicroMega (http://temi.repubblica.it/micromega-online/coronavirusmetafore-di-guerra-e-confusione-di-concetti/; data di ultima consultazione: 25.2.21).

Brambilla L. (2020): Donne e Covid-19. L'educazione sociale di una fatica indicibile. Studi sulla Formazione, 23(1), pp. 107-115.

Burgio G. (2029): L'eteronormatività come orizzonte. Bullismo omofobico e maschilità in adolescenza. In F. Dello Preite (a cura di): Femminicidio, violenza di genere e globalizzazione. Lecce: Pensa MultiMedia, pp. 55-68.

Cagnolati A., Pinto Minerva F., Ulivieri S. (a cura di) (2013): Le frontiere del corpo. Mutamenti e metamorfosi. Pisa: ETS.

Cambi F. (2001): Intercultura: fondamenti teorici. Roma: Carocci.

Cantarella E. (2019): Gli inganni di Pandora. L'origine delle discriminazioni di genere nella Grecia antica. Milano: Feltrinelli.

Caraveo P. (2017): Uomini e donne: stessi diritti? Roma: Castelvecchi.

Caritas Italiana (2020): Gli anticorpi della solidarietà. Rapporto 2020 su povertà ed esclusione sociale in Italia (http://s2ew.caritasitaliana.it/materiali/Rapporto_Caritas_2020/Report_CaritasITA_2020.pd; data di ultima consultazione: 25.2.21)

Cirillo L. (a cura di) (2018): Se il mondo torna nomo. Le donne e la regressione in Europa. Roma: Alegre.

Columba P. (2018): Il femminismo è superato. Falso! Roma-Bari: Laterza.

Commissione Europea (2013): Obiettivi di Barcellona. Lo sviluppo dei servizi di cura della prima infanzia in Europa per una crescita sostenibile e inclu- 
siva. COM/2013/0322 final (https://ec.europa.eu/transparency/regdoc/ rep/1/2013/IT/1-2013-322-IT-F1-1.Pdf; data di ultima consultazione: 7.3.21).

Commissione Europea (2020): Un'Unione dell'uguaglianza: la strategia per la parità di genere 2020-2025. COM/2020/152 final (https://eur-lex.europa. eu/legal-content/IT/TXT/?uri=CELEX\%3A52020DC0152, data di ultima consultazione: 7.3 .21 .

Contini M. (a cura di) (2010): Molte infanzie molte famiglie. Interpretare i contesti in pedagogia. Roma: Carocci.

Contini M., Ulivieri S. (a cura di) (2010): Donne, famiglia, famiglie. Milano: Guerini.

D'Addio A.C. (2020): Il rischio di ampliamento delle differenze di genere e i limiti della conciliazione. In Dipartimento per le politiche della famiglia (a cura di): L'impatto della pandemia di Covid-19 su natalità e condizione delle nuove generazioni. Primo rapporto del Gruppo di esperti "Demografia e Covid-19 (http://famiglia.governo.it/media/2192/rapporto-gruppo-demografia-e-covid19_1412020.pdf; data di ultima consultazione: 7.3.21).

Del Boca D., Oggero N., Profeta P., Rossi M.C., Villosio C. (2020): Prima, durante e dopo il Covid: disuguaglianze in famiglia (http://www.ingenere.it/ articoli/prima-durante-dopo-covid-disuguaglianze-famiglia; data di ultima consultazione: 28.2.21).

Dello Preite F. (2018): Educazione di genere e formazione dei talenti. Nuove sfide per la scuola del XXI Secolo. Formazione E Insegnamento, n. 2, pp. 73-79.

Dello Preite F. (a cura di) (2019): Femminicidio, violenza di genere e globalizzazione. Lecce: Pensa MultiMedia.

Di Sandro E. (2020): Nonne, nonni e nipoti. Rapporti intergenerazionali e prospettive pedagogiche. Pisa: ETS.

Dugarova E. (2020): Unpaid Care Work in Times of the COVID-19 Crisis: Gendered Impacts, Emerging Evidence and Promising Policy Responses (http:// www.bollettinoadapt.it/wp-content/uploads/2020/11/Duragova.Paper.pdf; ultima consultazione: 9.3.21).

EIGE (2021): The Covid-19 Pandemic and Intimate Partner Violence against Women in the EU (https://eige.europa.eu/news/covid-19-derails-genderequality-gains; data di ultima consultazione: 7.3.21).

FRA (2014): Violenza contro le donne: un'indagine a livello di Unione europea. Panoramica dei risultati (https://fra.europa.eu/sites/default/files/fra2014-vaw-survey-at-a-glance_it.pdf; data di ultima consultazione: 5.3.21).

Gigli A. (2010): Nuove donne per nuove famiglie. In M. Contini, S. Ulivieri (a cura di): Donne, famiglia, famiglie. Milano: Guerini.

Iori V. (a cura di) (2014): Fare la differenza. Analisi e proposte di gender management. Milano: FrancoAngeli.

Istat (2020a): Le statistiche dell'Istat sulla povertà (https://www.istat.it/it/files//2020/06/REPORT_POVERTA_2019.pdf; data di ultima consultazione: 25.2 .21 ). 
Istat (2020b): Violenza di genere al tempo del Covid-19: le chiamate al numero verde 1522 (https://www.istat.it/it/files//2020/05/Stat-today_Chiamate-numero-antiviolenza.pdf; data di ultima consultazione: 25.2.21).

Istat (2020c): Il numero di pubblica utilità 1522 durante la pandemia (periodo marzo-ottobre 2020; https://www.istat.it/it/archivio/250804\#: :text=Il\%20 servizio \%201522\%20svolge \%20un,viene \%20inviato \%20ad \%20un \%20 CAV.; data di ultima consultazione: 25.2.21).

Istat (2021a): Occupati e disoccupati (https://www.istat.it/it/archivio/253019; data di ultima consultazione: 28.2.21).

Istat (2021b): Proposta di "piano nazionale di ripresa e resilienza" (https://www. istat.it/it/archivio/253108; ultima consultazione: 28.2.21).

Lipperini L., Murgia M. (2013): L’ho uccisa perché l'amavo. Falso! Roma-Bari: Laterza.

Loiodice I. (a cura di) (2020): Ripensare le relazioni intergenere. Studi e riflessioni per la prevenzione e la lotta alla violenza contro le donne. Bari: Progedit.

Lopez A.G. (a cura di) (2017): Decostruire l'immaginario femminile. Percorsi educativi per vecchie e nuove forme di condizionamento culturale. Pisa: ETS.

Macinai E. (2020, 20 novembre): Trent'anni di diritti dell'infanzia e dell'adolescenza, un cammino non ancora compiuto. la Repubblica (https:// roma.repubblica.it/dossier-adv/eccellenze-lazio/2020/11/23/news/ trent_anni_di_diritti_dell_infanzia_e_dell_adolescenza_un_cammino_ non_ancora_compiuto-275434799/, e https:/www.siped.it/wp-content/ uploads/2020/11/2020-10-17-SIPED-Polenghi-Presidente-la-Repubblica-20.11.2020.pdf; data di ultima consultazione: 3.3.21).

Mancaniello R. (2020): Adolescenti al tempo del Covid-19: una riflessione sul significato di vivere "attimi della catastrofe adolescenziale" in uno spaziotempo negato, nella separazione corporea dal gruppo dei pari e in una relazione scolastica digitale. Studi sulla Formazione, 23(1), pp. 21-51.

Margiotta U. (2018): La formazione dei talenti. Tutti i bambini sono un dono, il talento non è un dono. Milano: FrancoAngeli.

Marone F. (a cura di) (2016): Raccontare le famiglie. Legami, società, educazione. Lecce-Rovato (Bs): Pensa MultiMedia.

Morin E. (2020): Cambiamo strada. Le 15 lezioni del Coronavirus. Trad. it. Milano: Raffaello Cortina, 2020.

Musi E. (2019): Le forme della discriminazione nei luoghi di lavoro. In F. Dello Preite (a cura di), Femminicidio, violenza di genere e globalizzazione. Lecce: Pensa MultiMedia, pp. 155-166.

Murgia A., Poggio B. (a cura di) (2011): Padri che cambiano. Sguardi interdisciplinari sulla paternità contemporanea tra rappresentazioni e pratiche quotidiane. Pisa: ETS.

Palmonari A. (2001): Gli adolescenti. Né adulti, né bambini alla ricerca della propria identità. Bologna: il Mulino.

Perillo P. (2016): Il "paternage" e le memorie familiari. Un ambito di intervento per il lavoro educativo con gli adulti. In F. Marone (a cura di): Raccontare le 
famiglie. Legami, società, educazione. Lecce-Rovato (BS): Pensa MultiMedia, pp. 381-422.

Priulla G. (2013): C'è differenza. Identità di genere e linguaggi: storie, corpi, immagini e parole. Milano: FrancoAngeli.

Saraceno C. (2016): Mamme e papà. Gli esami non finiscono mai. Bologna: il Mulino.

Saraceno C. (2017): L'equivoco della famiglia. Bari-Roma: Laterza.

Sartori F. (2009): Differenze e disuguaglianze di genere. Bologna: il Mulino.

Schimmenti V. (a cura di) (2005): Donne e professione. Percorsi delle femminilità contemporanea. FrancoAngeli: Milano.

Senato della Repubblica - XVIII legislatura (2020): Commissione Parlamentare d'inchiesta sul femminicidio, nonché su ogni forma di violenza di genere. Misure per rispondere alle problematiche delle donne vittime di violenza, dei centri antiviolenza, delle case rifugio e degli sportelli antiviolenza e antitratta nella situazione di emergenza epidemiologica da Covid-19. Doc. XXII-bis n. 1 (http://senato.it/service/PDF/PDFServer/DF/350725.pdf; data di ultima consultazione: 3.3 .21 ).

Silva C. (a cura di) (2017): Educazione e cura dell'infanzia nell'Unione Europea. Pisa: ETS.

Tomarchio M., Ulivieri S. (a cura di) (2015): Pedagogia militante. Diritti, culture, territori. Pisa: Pisa.

Ulivieri S. (1994): I silenzi sociali: l'infanzia, i giovani, le donne. Una storia ai margini. In F. Cambi, S. Ulivieri (a cura di): I silenzi dell'educazione. Studi storico-pedagogici. Scandicci (Fi): La Nuova Italia, pp. 53-71.

Ulivieri S. (a cura di) (2014): Corpi violati. Condizionamenti educativi e violenze di genere. Milano: FrancoAngeli.

Vegetti Finzi S., Battistin A.M. (2000): L'età incerta. I nuovi adolescenti. Milano: Mondadori.

Zanatta A.L. (2011): Nuove madri e nuovi padri. Essere genitori oggi. Bologna: il Mulino. 